\title{
Research on the Theory and Practice of Higher Mathematics Concept Map
}

\author{
Nan Wang \\ Department of foundation \\ Beijing Polytechnic, \\ Beijing, China \\ 85454456@qq.com
}

\begin{abstract}
Higher mathematics is one of the most important required courses in Colleges and Universities, the content of the teaching logic is very strong and the mathematical concept is abstract. The traditional teaching methods are monotonous, and there is a gap between senior high school mathematics and higher mathematics, which makes the students acquire knowledge more passively. The concept map can display the concepts of more complex, complicated and easily confused concepts in higher mathematics, it can clarify the structure of the concept, and is conducive to the dissemination, understanding, memory and consolidation of knowledge. Concept map can be used as the strategy and method of teaching and learning in the teaching of advanced mathematics, it helps to train students' creative thinking, and can be used as a teaching evaluation tool. Concept map can promote the teaching effect and improve the learning efficiency, and has higher application value.
\end{abstract}

Keywords-Concept map; Theory; Practice; Higher mathematics

\section{INTRODUCTION}

Mathematical concepts have a strict logic from concrete to abstract, from special to general, from partial to whole. Learn mathematics concepts and the relationship between, can make students form a good cognitive structure of mathematics reasoning ability, logical thinking ability of students, and improve the ability of solving problems. The concept of advanced mathematics is more synthetical and abstract, and contains profound and rich dialectical thoughts. It is difficult for students to understand and grasp accurately, which is one of the reasons why they find it difficult to learn advanced mathematics. In addition, the difference between higher mathematics and elementary mathematics is very large, and many of the knowledge has been broken or not very closely linked, it also makes it difficult for students to study advanced mathematics. The mathematical concept map can help students understand the knowledge structure of higher mathematics, clarify the links between relevant knowledge and enhance their interest in advanced mathematics.

Concept map is a kind of network graphics to structure knowledge, all concepts related to the domain of knowledge are placed in a block diagram and then layered and joined together in terms of conceptual relationships, and indicate the relation between the concepts on the line, so as to indicate how the knowledge is connected. The mathematical concept map can clearly express the essential relationship between mathematical knowledge by graphic method, and can make implicit mathematical knowledge explicit [1].

The concept map is applied to the construction of basic knowledge, integrating old and new knowledge, building knowledge network, concentrating knowledge structure, and grasping knowledge as a whole. It can be applied to brainstorming, creative association, and some complicated and difficult to remember knowledge, which is conducive to thinking, communication and expression. Although concept maps represent concepts, they also reflect the emotional state of the creator during the construction of the conceptual map. Concept maps are both rational and clear, and they also permeate the emotional qualities of the Creator, it is good for memory, and easily arouses the pride of the founder, and arouses the subjective initiative of the learner. It plays a very important role in the teaching.

\section{CONCEPT MAP THEORY}

\section{A. The theoretical basis}

The theoretical basis of conceptual map is Ausubel's theory of cognitive assimilation learning, Professor Novak of the Cornell University and others developed the concept map as a tool for teaching and learning, based on Ausubel's theory of cognitive assimilation learning, and apply it to practice teaching. He first applied it to the study of abstract concepts such as energy, cells, and evolution, later it was discovered that concept maps could also be applied to teaching design and to help students make meaningful learning, which led to a more in-depth study of concept maps.

Novak defines the concept map in the book "learning how to study ", Novak in "learning to learn" a Book concept map defined as: "the concept map is the tool to organize and represent knowledge, is a scientific proposition displayed the meaning connection between concepts, and with concrete examples to illustrate the spatial network structure linked to all the basic concepts of organic." 


\section{B. Structure feature}

Concept map is a graphical representation of the relationship between knowledge and knowledge, as well as the representation of visualization of thinking. A concept map is usually made up of nodes, links, and related text annotation [2]. Nodes are usually represented by geometric shapes, patterns, text, etc. each node represents a concept, usually in the same level, with the same symbol or graph. Links are used to represent meaningful relationships between different nodes, often using various lines or arrows to link different nodes, which express the degree of understanding of the concept of the composition. Text annotation can represent relationships between concepts on different nodes, or elaborate on concepts on nodes, or on the entire diagram.

\section{The general steps for creating concept maps:}

First, identify key concepts and other concepts associated with them, and list them as a list of key concepts. Then sort the concepts, the most conceptual and generic concepts are placed at the top (middle), and other concepts are downward (outward) in turn, the less general concept is located at the lower (outer) level, then the most specific concept is at the lowest (outermost) level, thus forming a hierarchy of concepts. Finally, link each link, connect each pair of related concepts with a line or arrow, and indicate what is or what is the relationship between them. In this way, concepts of the same field or different fields are connected by some related concepts.

\section{THE PRACTICAL ROLE OF THE CONCEPT MAP OF HIGHER MATHEMATICS}

The internal logic of higher mathematics knowledge is very strong, and the connection between knowledge points is very close. To enable students to seize the inner link between knowledge, applying concept map to integrate knowledge and form a good knowledge network is an effective method.

In the process of learning new concepts, the application of concept maps enables students to understand the occurrence, development process and extension of the existing concepts in the existing knowledge base, further clarify the correlation between concepts, it is beneficial for students to form systematic knowledge structure in their minds [3]. In reviewing the conceptual process, the application of concept maps can help students remember what they have learned before, students can explore the inner connection between concepts by arranging the concepts they have learned, the existing knowledge can be re integrated and a new structure of knowledge can be established. Finally, the relation between concepts is shown in the form of concept map, so as to realize the reorganization and generation of knowledge structure.

The application of mathematical concept map can fundamentally help students understand and grasp mathematical knowledge, and it will be of great help to the transfer of knowledge and the construction of knowledge as a whole. Besides the knowledge acquisition, students can develop their logical thinking ability and reasoning ability, as well as their ability to think independently and solve problems in the process of creating concept maps.

\section{A. An effective tool for classroom teaching design}

In the teaching of mathematics, the concept map can be used to visualize the teaching content, teaching theory and teaching experience that appear in the teacher's mind in visual form, it can help teachers organize the teaching content effectively. In the course of teaching, the teacher displays the process of knowledge integration clearly through conceptual map, which can change the cognitive style of students and make them see the relationship between concepts. Students are master of the whole knowledge frame, easier to understand the links and differences between new knowledge, and remember better than simple mechanical memory through concept maps [4].

The logic of advanced mathematics is very strong, in the process of teaching, teachers should guide students to grasp the internal relationship between knowledge and integrate new knowledge so as to form a good knowledge network. Creating concept maps is an effective method.

For example(Figure1), the limit, continuity, differentiability and differentiability of multivariate functions are more difficult for students to master. It is easier to understand the following concept maps, so that it can achieve twice the result with half the effort.

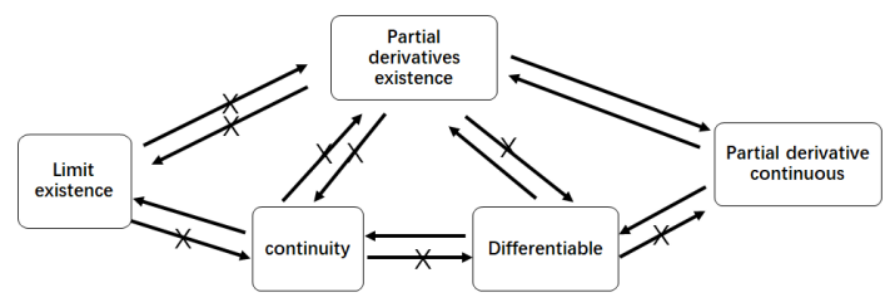

Fig. 1 Concept map

\section{B. Improve the teaching level of Teachers}

The mathematical concept map shows the close connection of mathematical knowledge with rigorous hierarchical relations and connection certainty. From mathematical concept maps, it is easy to identify the key concepts in teaching and the specific relationships between concepts. With the key concepts and new concepts as the core, the teaching process is designed, which can help the math teachers easily highlight the key points of teaching, break through the teaching difficulties, and complete the teaching design efficiently. The teacher can represent the new knowledge that students need to learn by using a mathematical concept map, and then design a teaching process based on a conceptual map containing new mathematical knowledge. By making mathematical concept map, it can help teachers to grasp the structure of teaching knowledge as a whole, and the key and difficult points are clear at a glance.

Conversely, teachers can also use concept maps to represent instructional design, In the process of turning teaching design into the concept map, not only can deepen teachers' understanding of the teaching content, but also familiar with the specific process of teaching activities, so as to help the teachers to grasp the whole teaching process. The teacher carries on the teaching according to the concept map, in 
the teaching process may help to carry on the goal instruction to student's mathematics study, thus enables the teaching activity to carry on according to the teaching design highly effective and orderly [5]. Finally, through the teaching process and students' learning effect, we can reflect on the teaching design concept map, and find out the problems existing in the teaching design to improve, and constantly improve their teaching level.

\section{Help students learn math}

Applying mathematical concept maps to mathematics learning can effectively help students learn mathematics. Will the teacher students to learn new knowledge into mathematical concept map is presented to students, students through observation of mathematical concept map to quickly identify the relationship between concepts, actively connect the concepts in the mind with the new mathematical concept maps, so as to help students understand the new knowledge.

Students can also help themselves to understand new knowledge by creating their own mathematical concept maps. Especially in the mathematical chapters with more concepts, the concept map is used to present the knowledge in a holistic way, which is conducive to the understanding, memory and consolidation of knowledge, and the improvement of learning efficiency [6]. The students in the process of creating the concept map, through positive thinking, to find out the relationship between the concept of need from the existing knowledge structure and its related concepts, so that students can achieve the purpose of the review to consolidate the knowledge learned.

For example (Figure 2), let students make concept maps using the limits of calculus as key concepts, students need to understand the meaning of the limit, including the sequence, the limit meaning of the function, familiar with the limit of the algorithm and some special criteria, analysis of the relationship between the various concepts. Students further explore the relevant concepts of differences and relations between the process, in fact, requires students to think at the conceptual level, belonging to advanced thinking. This process of creating concept maps can promote the learning of new knowledge, also can deepen students' deep understanding of the knowledge, so as to understand the relevance of mathematical knowledge as a whole, stimulate students' interest in learning, promote the development of thinking, and improve the learning efficiency of students [7].

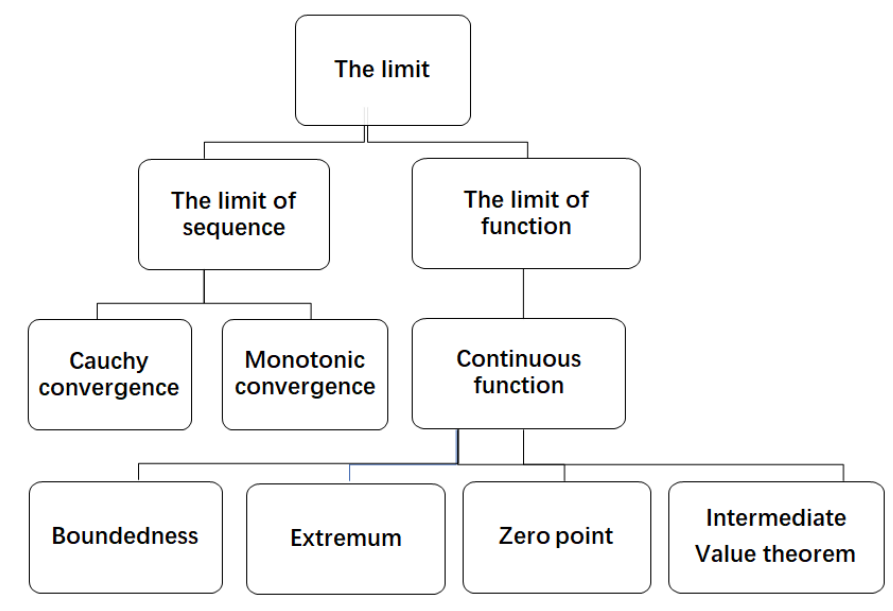

Fig. 2 Concept map of limit

\section{Perfect mathematics teaching evaluation}

The mathematics concept map provides a new way for teachers to evaluate students' mathematics learning results. Teachers can check out the correct or incorrect understanding of concepts and their depth of conceptual understanding through the concept maps produced by students. And by making students' mathematical concept map in nodes and connected word check, can understand the degree of knowledge structure and further clarify the related concepts in the minds of students, so as to accurately evaluate the students' mastery of knowledge and development of mathematical thinking level.

As a way of evaluating the effect of mathematics learning, mathematics concept map has the characteristics of quickness, simplicity and efficiency, and its evaluation effect is not achieved by traditional evaluation methods. Teachers can check the students mathematical concept map creation, can understand the degree of students' knowledge structure of mathematics, and found that there is insufficient or incorrect contact, targeted for correction or guidance to students [8]. As a way of evaluation, mathematics concept map can enrich and perfect the mathematical knowledge structure of students, so as to give full play to the process evaluation function of concept map, which is of positive significance to evaluate students' learning.

\section{THE INADEQUACY OF CONCEPT MAPS}

A mathematical concept map is a method of demonstrating the cognitive structure of mathematical knowledge. The introduction of mathematical concept map into higher mathematics teaching has some advantages, but at the same time, we should look at it rationally and rationally, and we cannot exaggerate the function of concept map. Students have grasped the relationship between concepts in the concept map, which does not mean that students have mastered all the knowledge involved in the concept map.

The concept map can show students a basic knowledge framework, the construction of the concept maps is the relationship between the concepts, just to help students in mathematics to solve the problem of sorting out the reliable 
ideas, it cannot construct method of mathematical problem solving, mathematics experiments etc. Therefore, mathematical concept map requirements on the content of classroom teaching is not every lesson applicable, sometimes simply to change a teaching method and insist on using the concept map can backfire, does not comply with the law of education.

\section{CONCLUSIONS}

The holistic and structured visual representation of conceptual maps provides learners with a structured learning space. The concept map information stored in the way, just as our brain itself connected by a node of storage, can appear between the content and the content of a clear link, can effectively help learners overall understanding of knowledge.

In the higher mathematics education, rational use of concept map can help students form a solid framework for the knowledge in books, but also makes the students understand the knowledge more deeply, to keep the knowledge on more long-term memory. Through the production of mathematical concept map, we can better cultivate students' logical thinking and creativity, and make the students' thinking more clear and active, and help them to understand the relevant knowledge intuitively. In higher mathematics teaching, it is a very effective teaching tool to make full use of concept map, establish network system of concepts and deepen concept teaching, it can improve the teaching quality of colleges and universities, and the mathematical concept map has higher application value.

\section{REFERENCES}

[1] Karoline Afamasaga-Fuata: Concept Mapping in Mathematics [M]. New York, USA: Springer, 2009

[2] Joseph.D.Novak(2000):The Theory Underly-ing Concept Maps and To Construct Them, In-ternet.

[3] Barbara Guzzetti \& Cynthia Hynd(1998) Perspectives on Conceptual Change Published by Lawrence Erlbaum Associates,London

[4] Douglas A Grouws. Handbook of Research on Mathematics Teaching and Learning $[\mathrm{M}]$. New York: Macmillan Publishing Company, 1992.

[5] SCANLON D.Interactive Semantic Mapping:on Interactive Approach tonhancing LD Students' Content Area Comprehension [J].Learning Disability Research and Practice,1992,7(3):142-146.

[6] Guo-Xiang Gui. How to optimize the teaching of advanced mathematics [J]. The Science Education Article Collects, 2014,(13)(In Chinese)

[7] Ying Li. Review and application of concept map research [J]. Journal of Shanxi Datong University (SOCIAL SCIENCE EDITION),2011,(6) (In Chinese)

[8] Xiao-Yong Hu, Yong Wang, Mei Rong. Concept map teaching and training course [M]. Nanjing, Nanjing Normal University press, 2008. (In Chinese) 